\title{
LARGE AXIALLY SYMMETRIC STRETCHING OF A NONLINEAR VISCOELASTIC MEMBRANE
}

\author{
ALAN S. Wineman $\dagger$ \\ Department of Engineering Mechanics, University of Michigan, Ann Arbor, Michigan 48104
}

\begin{abstract}
Two problems of large planar axisymmetric deformations of an annular membrane consisting of a nonlinear viscoelastic material are solved, one with prescribed deformation at the outer boundary and one with prescribed force. These problems serve as examples to illustrate the extension to a class of viscoelastic membrane problems of a formulation of the corresponding elastic membrane problem suggested by Yang [1], which is especially convenient for numerical solution. The formulation uses radial and circumferential stretch ratios as dependent variables, which in the present case are found by solving a system of first order nonlinear partial differentialintegral equations. The numerical procedure is such that at each time step, the problem is equivalent to a system of first order nonlinear ordinary differential equations for the current stretch ratios. This system is then integrated by the same numerical procedure as in the corresponding elastic problem.
\end{abstract}

\section{INTRODUCTION}

A NUMBER of problems involving large axially symmetric deformations of initially plane membranes formed of incompressible nonlinear elastic solids have recently been reformulated and solved numerically by Yang [1]. The usual formulation defines a radial deformation function which satisfies a second order nonlinear ordinary differential equation. By introducing the radial and circumferential stretch ratios as dependent variables, Yang reduced this equation to a system of two linear first order equations whose numerical solution is more readily obtained. This has the further advantage that boundary conditions are more easily stated in terms of these stretch ratios than in terms of the deformation function, while stresses and the deformation function are readily calculated.

Each of these axially symmetric problems involving an elastic membrane suggests a corresponding problem of large quasistatic motions of a viscoelastic membrane by letting the membrane now be viscoelastic and the applied axially symmetric stresses vary with time. The formulation of the latter problem is facilitated by the observation that the same kinematical assumptions regarding the equilibrium configuration of the elastic membrane can also be made about each of the sequence of configurations assumed by the viscoelastic membrane during its motion. A time dependent radial deformation function is thus defined which satisfies a second order nonlinear partial differential-integral equation. Because of this similarity in kinematics, the advantages of the stretch ratio formulation can be extended to the viscoelasticity problem. The purpose here is to illustrate this approach for a specific problem concerning an initially plane annular membrane of an incompressible isotropic nonlinear viscoelastic solid. This formulation leads to a system of two first order nonlinear partial differential-integral equations for the radial and circumferential stretch ratios as functions of time and undeformed radius. Numerical

Associate Professor. 
solutions are then obtained for the two point boundary value problems in which the inner hole is stress free and either time dependent stress or radius is prescribed at the outer boundary. The numerical procedure is such that at each time step one obtains a first order system of differential equations for the corresponding stretch ratios, as in the elasticity case.

The governing equations are developed in Section 2. Although the general method does not depend on the form of constitutive equation used to represent viscoelastic behavior, for convenience the governing field equations are derived using a general single integral constitutive equation. In the elastic problem, the condition that radial stress vanish at a boundary implies an algebraic relation among the stretch ratios. This same relation is shown to hold for any viscoelastic constitutive equation. In order to obtain numerical results, a specific form of constitutive equation must be used. The one selected is discussed in Section 3. The details of the numerical method are given in Section 4 and the numerical results are discussed in Section 5.

The stretch ratio formulation has been extended by Yang et al. (see [2, 3]), to a variety of problems involving axially symmetric deformations of elastic membranes initially in the shape of surfaces of revolution. Such problems also suggest corresponding viscoelasticity problems. Just as is illustrated here, because of the similarity in kinematics, the approach to the elasticity problem can be immediately applied to the viscoelasticity case. Thus, although there appears to be no "correspondence theorem" proving direct operational methods for obtaining viscoelasticity solutions from elasticity solutions as in the linear theories, for the class of problems considered here there is outlined a "correspondence procedure".

\section{FORMULATION}

The undeformed annular membrane has inner radius $a$, outer radius $b$ and uniform thickness $h$, where $h / a \ll 1$. Let the origin of a cylindrical polar coordinate system coincide with the center of the hole and its plane $z=0$ coincide with the middle plane of the sheet. For a sufficiently thin sheet, the stresses on planes $z=$ const. can be neglected and the deformations under consideration can be described by the mapping [4]

$$
\rho=\rho(r, t), \quad \theta=\Theta, \quad \zeta=z \lambda_{3}(r, t)
$$

where $(r, \Theta, z)$ are coordinates in the undeformed state and $(\rho, \theta, \zeta)$ are coordinates at time $t$. The deformation is such that at each time $t$, the coordinate directions are principal directions. The stretch ratios in the radial and circumferential directions are, respectively,

$$
\begin{aligned}
& \lambda_{1}=\frac{\partial \rho}{\partial r}(r, t), \\
& \lambda_{2}=\frac{\rho(r, t)}{r},
\end{aligned}
$$

while that in the $z$-direction, $\lambda_{3}$, is determined through the incompressibility condition

$$
\lambda_{1} \lambda_{2} \lambda_{3}=1
$$

For notational convenience, let $\lambda_{i}=\lambda_{i}(t), \lambda$ denote the triplet $\left(\lambda_{1}, \lambda_{2}, \lambda_{3}\right)$ and $\lambda(\tau)$ denote the triplet $\left(\lambda_{1}(\tau), \lambda_{2}(\tau), \lambda_{3}(\tau)\right)$. 
The strain invariants are

$$
\begin{aligned}
& I_{1}=\lambda_{1}^{2}+\lambda_{2}^{2}+\lambda_{3}^{2}, \\
& I_{2}=\lambda_{1}^{2} \lambda_{2}^{2}+\lambda_{2}^{2} \lambda_{3}^{2}+\lambda_{3}^{2} \lambda_{1}^{2}, \\
& I_{3}=\lambda_{1} \lambda_{2} \lambda_{3}=1 .
\end{aligned}
$$

For future reference, it will be useful to note that the stretch ratios satisfy the following compatibility condition for each $t \geq 0$ :

$$
\frac{\partial \lambda_{2}}{\partial r}=\frac{\lambda_{1}-\lambda_{2}}{r} .
$$

The non-zero components of stress, per unit deformed area, are in the radial and circumferential directions and are denoted, respectively, by $\sigma_{1}$ and $\sigma_{2}$. Their resultants, measured per unit current length in the appropriate direction in the middle plane of the membrane, are defined by

$$
T_{1}=h \lambda_{3} \sigma_{1}, \quad T_{2}=h \lambda_{3} \sigma_{2},
$$

where by (2.1), $h \lambda_{3}$ is the current thickness of the sheet. The equations governing the quasistatic motion are automatically satisfied in the $\Theta$ and $z$ directions. Taking into consideration thickness changes and ignoring the presence of body forces, the equation in the radial direction is

$$
\frac{\partial}{\partial \rho}\left(\rho T_{1}\right)=T_{2}
$$

For each time $t,(2.7)$ must be satisfied in the unknown region occupied by the membrane, $\rho(a, t) \leq \rho \leq \rho(b, t)$. It is convenient to regard all quantities of interest as functions of the initial coordinate $r$ and time $t$ since $r$ varies in the known initial region occupied by the membrane, $a \leq r \leq b$. Introducing the transformation (2.1) and then using (2.2), (2.3) and (2.6), equation (2.7) becomes for each $t \geq 0$,

$$
\frac{\partial}{\partial r}\left(\lambda_{3} \sigma_{1}\right)=\frac{1}{r} \frac{\lambda_{1} \lambda_{3}}{\lambda_{2}}\left(\sigma_{2}-\sigma_{1}\right)
$$

Define dimensionless variables as follows:

$$
\bar{r}=r / a, \quad \bar{\rho}=\rho / a, \quad \bar{b}=b / a, \quad \bar{t}=t / \tau_{R}, \quad \bar{\sigma}_{\alpha}=\sigma_{\alpha} /\left(h C_{0}\right),
$$

where $C_{0}$ is an elastic modulus and $\tau_{R}$ is a typical relaxation time. Because of their homogeneity in $r, \rho, \sigma_{\alpha}$ and $T_{\alpha}$, equations (2.2), (2.5), (2.7) and (2.8) may be regarded as expressed in terms of dimensionless variables with the bar notation dropped. There will be no loss in generality, then, in saying that at the inner radius $r=1$ and at the outer radius $r=b$.

Up to this point, the only difference between the present formulation and that for an elastic membrane is the presence of a time parameter $t$. This is entirely a consequence of the following two points: (1) because the problem is axisymmetric, the radial and circumferential directions are principal directions for all $t \geq 0$, (2) the assumed form of the deformation function (2.1) for the viscoelastic membrane at any time $t$ is the same as would be made for the equilibrium configuration of the elastic membrane. The principal stretch ratios defined in (2.2), relation (2.5) and the force balance equation (2.7) in terms of principal 
stresses, being derived by spatial operations at each time $t$, are therefore the same as in the elastic case. It follows that equation (2.8) arises in both the "elastic" and "viscoelastic" formulations. The two formulations differ, of course, through the way their respective constitutive equations relate stress, stretch ratios and time. However, when these latter equations are combined with (2.8), the elastic and viscoelastic formulations each obtains one equation for the principal stretch ratios. The second equation in either formulation is provided by (2.5). Thus, the use of principal stretch ratios as dependent variables in this viscoelastic membrane problem is a natural extension of their use in the corresponding elastic membrane problem in [1].

The remainder of the formulation will be carried out using a single integral constitutive equation to represent the behavior of a nonlinear incompressible isotropic solid. Coleman and Noll [5] have derived one such model for a theory of finite linear viscoelasticity using concepts of fading memory, while Pipkin and Rogers [6] have constructed a second model based on the presumed nonlinear response of a material to a series of step strain inputs. The Pipkin and Rogers constitutive equation will be used because it involves fewer relaxation functions. Its general form is

$$
\boldsymbol{\sigma}(t)=-p \mathbf{1}+\mathbf{F}(t)\left\{\mathbf{R}[C(t) ; 0]+\int_{0}^{t} \partial_{\mathbf{r}} \mathbf{R}[\mathbf{C}(\tau), t-\tau] \mathbf{d} \tau\right\} \mathbf{F}^{T}(t),
$$

where $\partial_{t}=\partial / \partial(t-\tau), p$ is the unknown scalar reaction to (2.3), $\mathbf{F}$ is the deformation gradient tensor, $\mathbf{C}=\mathbf{F}^{\mathbf{T}} \mathbf{F}$. $\mathbf{R}[\mathbf{C}, t]$ is the strain dependent tensorial relaxation function induced by a single step strain history, $\mathbf{C}(\tau)=\mathbf{C}^{*}, \tau \geq 0$ and has form

$$
\mathbf{R}=\phi_{0} \mathbf{1}+\phi_{1} \mathbf{C}+\phi_{2} \mathbf{C}^{2},
$$

$\phi_{0}, \phi_{1}, \phi_{2}$ being scalar functions of $t$ and the invariants of $\mathbf{C}$.

Writing (2.9) and (2.10) in components with respect to the cylindrical coordinate system and determining $p$ from the condition that $\sigma_{3}=0$, the following expressions for $\sigma_{1}$ and $\sigma_{2}$ are obtained:

$$
\begin{aligned}
\sigma_{\alpha} & =\phi_{0}\left(\lambda_{\alpha}^{2}-\lambda_{3}^{2}\right)+\phi_{1}\left(\lambda_{\alpha}^{4}-\lambda_{3}^{4}\right)+\phi_{2}\left(\lambda_{\alpha}^{6}-\lambda_{3}^{6}\right) \\
& +\sum_{i=0}^{2} \int_{0}^{t} \partial_{t} \phi_{i}\left(\lambda_{\alpha}^{2} \lambda_{\alpha}^{2 i}(\tau)-\lambda_{3}^{2} \lambda_{3}^{2 i}(\tau)\right) \mathrm{d} \tau \quad(\alpha=1,2)
\end{aligned}
$$

The constitutive equation in (2.9) or (2.11) gives the stress components at a particle only if $\lambda_{i}(\tau)$ is the stretch ratio history for that particle. The stretch ratio history for a specific particle can be identified by letting $\lambda_{i}(\tau)$ depend on that particle's label, which is taken as its initial coordinates. Because of the axial symmetry of the problem all particles at the same initial radius have the same deformation history so $\lambda_{i}(\tau)$ depends only on the initial radius $r$. Note that this is also consistent with the use of $r$ as an independent variable in (2.8).

On substituting $\sigma_{1}$ from (2.11) into the left hand side of (2.8) and differentiating, one obtains terms involving $\partial \lambda_{1} / \partial r, \partial \lambda_{2} / \partial r, \partial \lambda_{3} / \partial r$. The latter two can be eliminated by using (2.5) and the following auxiliary result obtained by differentiating (2.3):

$$
\frac{\partial \lambda_{3}}{\partial r}=-\frac{1}{\lambda_{1} \lambda_{2}}\left(\frac{1}{\lambda_{1}} \frac{\partial \lambda_{1}}{\partial r}+\frac{1}{\lambda_{2}} \frac{\partial \lambda_{2}}{\partial r}\right)
$$


Evaluating the right hand side of (2.8) by means of (2.11) and rearranging terms, one obtains an equation of form

$$
\begin{aligned}
& \frac{\partial \lambda_{1}}{\partial r}\left\{F_{1}(\lambda)+\int_{0}^{t} G_{1}(\lambda ; \lambda(\tau) ; t-\tau) \mathrm{d} \tau\right\}+\int_{0}^{t} \frac{\partial \lambda_{1}(\tau)}{\partial r} G_{2}(\lambda ; \lambda(\tau) ; t-\tau) \mathrm{d} \tau \\
& =\frac{1}{r}\left\{F_{2}(\lambda)+\int_{0}^{t} G_{3}(\lambda ; \lambda(\tau) ; t-\tau) \mathrm{d} \tau\right\} .
\end{aligned}
$$

$F_{1}, F_{2}, G_{1}, G_{2}$ and $G_{3}$ depend on the details of the calculations and the form of the material parameters $\phi_{0}, \phi_{1}, \phi_{2}$. They will be given in the next section when a particular material is considered. On differentiating the product $\lambda_{3} \sigma_{1}$, both $\partial \lambda_{3} / \partial r$ and the derivative of $\sigma_{1}$ give rise to the term $\partial \lambda_{1} / \partial r\{-\}$ in (2.12). The derivative of the integrals in $\sigma_{1}$ also leads to the integral term involving the history of $\partial \lambda_{1} / \partial r(\tau), \tau \geq 0$. Thus, a Volterra integral operator which is linear in $\partial \lambda_{1} / \partial r$, such as appears on left hand side of (2.12), will be present for any integral constitutive equation. The numerical solution utilizes this property.

Equations (2.5) and (2.12) form a system of two nonlinear partial differential-integral equations of Volterra type for $\lambda_{1}(r, t)$ and $\lambda_{2}(r, t)$. The system must now be supplemented by appropriate boundary data. The condition at the inner boundary is that the hole be stress free, $\sigma_{1}(1, t)=0, t \geq 0$. From $(2.11)$ it is seen that this condition is satisfied if $\lambda_{1}(1, t)=\lambda_{3}(1, t), t \geq 0$, or by $(2.3)$,

$$
\lambda_{1}(1, t)=\lambda_{2}^{-\frac{1}{2}}(1, t), \quad t \geq 0
$$

This is related to the more physically meaningful deformation function by (2.2),

$$
\rho(1, t)=\lambda_{2}(1, t)
$$

In concept, specifying the growth of the inner hole $\rho(1, t)$ gives by $(2.13)$ and $(2.14)$ boundary data on the stretch ratios. Since the spatial aspect of (2.5), (2.12) defines essentially an initial value problem in $r$, one can solve for the stretch ratios to any desired radius and time. It is then an easy matter to calculate $\rho(r, t), \sigma_{1}(r, t), \sigma_{2}(r, t)$ or, more physically, how the membrane must be stretched so that the inner hole grows as prescribed. In a practical situation, however, it is more likely that conditions would be specified at the outer radius. For this reason, problems of the following type are considered :

(a) Relaxation problem - the increase of the outer radius, $\rho(b, t)$, is specified. By $(2.2)$, this is equivalent to prescribing $\lambda_{2}(b, t)=\lambda^{*}(t)$.

(b) Creep problem-the radial stress at the outer boundary, $\sigma_{1}(b, t)$, is specified. In the particular arrangement considered, there is a series of wires distributed uniformly around the outer circumference, directed radially outward, strung over pulleys and having fixed weights on their ends, as shown in Fig. 1. The radial stress is then determined by the condition that the total weight equal $2 \pi \rho\left(h \lambda_{3}\right) \sigma_{1}$ evaluated at $r=b$. In this case, $F^{*}(t)=$ $\rho(b, t) \lambda_{3}(b, t) \sigma_{1}(b, t)$ is specified.

In both cases, the numerical method must determine $\lambda_{2}(1, t)$ so that the integration of (2.5), (2.12) and (2.13) leads the prescribed condition at $r=b$.

Finally, it is worth pointing out that the boundary condition $\sigma_{1}(1, t)=0, t \geq 0$, will be satisfied by (2.13) for any constitutive equation for an incompressible isotropic solid. 


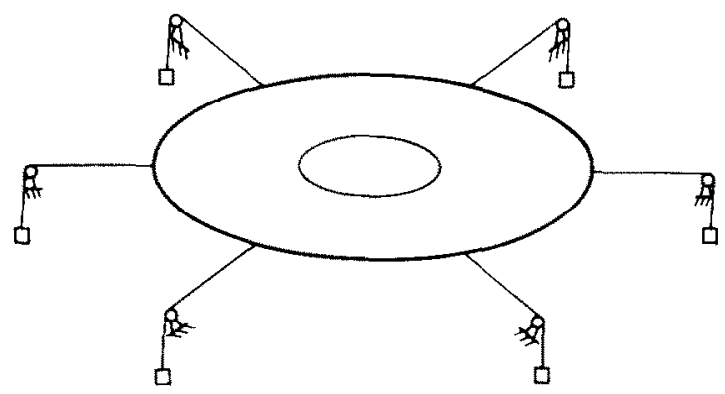

FIG, 1. Loading configuration for the creep problem (force boundary condition).

Wineman and Pipkin [7] have shown that the most general form for such a constitutive equation is:

$$
\sigma=-p \mathbf{l}+\mathbf{F}\left\{\sum_{i=1}^{5} R^{(i)}\left[\mathbf{G}^{(i)}\left(\tau_{1}, \tau_{2}, \ldots, \tau_{i}\right) ; I\right]\right\} \mathbf{F}^{T},
$$

where $R^{(i)}$ is a general functional which is linear in the tensor product $\mathbf{G}^{(i)}\left(\tau_{1}, \tau_{2}, \ldots, \tau_{i}\right)=$ $\mathbf{C}\left(\tau_{1}\right) \mathbf{C}\left(\tau_{2}\right) \ldots \mathbf{C}\left(\tau_{i}\right), 0 \leq \tau_{i} \leq t$, and depends also on a set of invariants $I$. Such functionals may contain distributions which give rise to the non-integral terms in (2.9). Calculating the tensors $\mathbf{F}$ and $\mathbf{C}$ for deformation (2.1) and then expressing (2.15) with respect to cylindrical coordinates on the middle plane of the membrane, one obtains

$$
\sigma_{\alpha}=-p+\sum_{i=1}^{5} R^{(i)}\left(\lambda_{\alpha}^{2} \lambda_{\alpha}^{2}\left(\tau_{1}\right) \lambda_{\alpha}^{2}\left(\tau_{2}\right) \ldots \lambda_{\alpha}^{2}\left(\tau_{i}\right) ; n, \quad(\alpha=1,2,3) .\right.
$$

Using the condition $\sigma_{3}=0$ to determine $p$ and then the linearity of the functionals in their tensorial arguments, one obtains:

$$
\sigma_{1}=\sum_{i=1}^{5} R^{(i)}\left(\lambda_{1}^{2} \lambda_{1}^{2}\left(\tau_{1}\right) \ldots \lambda_{1}^{2}\left(\tau_{i}\right)-\lambda_{3}^{2} \lambda_{3}^{2}\left(\tau_{1}\right) \ldots \lambda_{3}^{2}\left(\tau_{i}\right) ; I\right) .
$$

The condition $\sigma_{1}(t)=0, t \geq 0$, is satisfied if $\lambda_{1}(t)=\lambda_{3}(t), t \geq 0$, because the linearity property of the functionals implies $R^{(i)}(0 ; I)=0$.

\section{A SPECIFIC MATERIAL}

Pipkin and Rogers [6] do not present any specific forms for the strain dependent relaxation functions $\phi_{i}$ appearing in (2.9), (2.10). Since the present purpose is to illustrate a method of approach for treating viscoelastic membrane problems, $\phi_{0}, \phi_{1}, \phi_{2}$ are chosen for both analytical convenience and to represent general features of a nonlinear viscoelastic solid.

In particular, the constitutive equation (2.9), for single step strain inputs was chosen to combine features of the Mooney model of nonlinear elasticity and the standard solid of linear viscoelasticity. Written with respect to principal directions,

$$
\sigma_{i}=-p+\left[(1+\alpha I) \lambda_{i}^{2}-\alpha \lambda_{i}^{4}\right] \hat{R}(I ; t),
$$


where $I=I_{1}$ defined by (2.4) and $\alpha$ is a dimensionless constant. Relaxation properties are contained in the coefficient $\hat{R}(I ; t)$ which has a single exponential term analogous to the relaxation function for the standard solid of the linear theory. Letting $C_{0}$ denote the initial modulus, $C_{\infty}$ the residual modulus, and $\gamma=C_{\infty} / C_{0}$,

$$
\begin{aligned}
\hat{R}(I, t) & =C_{0}\left((1-\gamma) \mathrm{e}^{-p(I) t}+\gamma\right) \\
& =C_{0} C[I, t] .
\end{aligned}
$$

$p(I)^{-1}$ represents a strain dependent relaxation time with

$$
p(I)=\frac{1}{\tau_{R}}(1+\beta(I-3)),
$$

where $\beta$ is a positive dimensionless constant. The constant $\tau_{R}$ represents the relaxation time in the linearized theory since for small deformations $I \approx 3$.

Note that for both the initial response and the long time residual response, the coefficient $C(I ; t)$ reduces to a constant and (3.1) becomes exactly the constitutive equation for a Mooney material. In fact, in combining (3.1)-(3.3) with (2.9), this is also seen to be the case for transient stretch histories which approach fixed states as $t \rightarrow \infty$. The form of $p(I)$ is such that as the stretch ratios increase, the relaxation time decreases. Some authors suggest that the amount of stretching does not influence relaxation time [8]. On the other hand, others allow for this dependence but in a more complicated model than considered here $[9,10]$. By comparing calculations with $\beta=0$ and $\beta>0$, the influence of this effect on the behavior of the membrane can be assessed.

When (3.1)-(3.3) are substituted into (2.9), the stress-stretch ratio history expressions specialize as follows,

$$
\begin{aligned}
\sigma_{1}(t) / C_{0}= & \left(\lambda_{1}^{2}-\lambda_{3}^{2}\right)\left(1+\alpha \lambda_{2}^{2}\right) \\
& +\int_{0}^{t} \partial_{t} C[I(\tau) ; t-\tau]\left\{\left[\lambda_{1}^{2}-\lambda_{3}^{2}\right]\left[1+\alpha \lambda_{2}^{2}(\tau)\right]+\alpha\left[\lambda_{1}^{2} \lambda_{3}^{2}(\tau)-\lambda_{1}^{2}(\tau) \lambda_{3}^{2}\right]\right\} \mathrm{d} \tau, \\
\sigma_{2}(t) / C_{0}= & \left(\lambda_{2}^{2}-\lambda_{3}^{2}\right)\left(1+\alpha \lambda_{1}^{2}\right) \\
& +\int_{0}^{t} \partial_{t} C[I(\tau) ; t-\tau]\left\{\left[\lambda_{2}^{2}-\lambda_{3}^{2}\right]\left[1+\alpha \lambda_{1}^{2}(\tau)\right]+\alpha\left[\lambda_{2}^{2} \lambda_{3}^{2}(\tau)-\lambda_{2}^{2}(\tau) \lambda_{3}^{2}\right]\right\} \mathrm{d} \tau .
\end{aligned}
$$

The specific form of the functions in the partial differential-integral equation (2.12) corresponding to this model are:

$$
\begin{aligned}
& F_{1}=\left(\lambda_{1}^{4} \lambda_{2}^{2}+3\right)\left(1+\alpha \lambda_{2}^{2}\right) \\
& F_{2}=\frac{\lambda_{1}\left(\lambda_{2}-\lambda_{1}\right)}{\lambda_{2}}\left[\left(\lambda_{1}^{3} \lambda_{2}^{3}+3\right)+\alpha\left(\lambda_{1} \lambda_{2}+\lambda_{1}^{2}+\lambda_{2}^{2}+\lambda_{1}^{4} \lambda_{2}^{4}\right)\right] \\
& G_{1}=\partial_{t} C[I(\tau) ; t-\tau]\left\{\left(\lambda_{1}^{4} \lambda_{2}^{2}+3\right)\left(1+\alpha \lambda_{2}^{2}(\tau)\right)+\alpha\left(\frac{\lambda_{1}^{4} \lambda_{2}^{2}}{\lambda_{1}^{2}(\tau) \lambda_{2}^{2}(\tau)}+3 \lambda_{1}^{2}(\tau)\right)\right\} \\
& G_{2}=G_{2}^{\prime} \partial_{t} C[I(\tau) ; t-\tau]+G_{2}^{\prime \prime} \partial_{t} \frac{\partial C}{\partial I}(I(\tau) ; t-\tau) \\
& G_{3}=G_{3}^{\prime} \partial_{t} C[I(\tau) ; t-\tau]+G_{3}^{\prime \prime} \partial_{t} \frac{\partial C}{\partial I}(I(\tau) ; t-\tau)
\end{aligned}
$$




$$
\begin{aligned}
G_{2}^{\prime}= & -2 \alpha \frac{\lambda_{1}}{\lambda_{1}(\tau)}\left(\frac{\lambda_{1}^{4} \lambda_{2}^{2}}{\lambda_{1}^{2}(\tau) \lambda_{2}^{2}(\tau)}+\lambda_{1}^{2}(\tau)\right) \\
G_{2}^{\prime \prime}= & 2 \frac{\lambda_{1}}{\lambda_{1}(\tau)}\left[\left(\lambda_{1}^{4} \lambda_{2}^{2}-1\right)\left(1+\alpha \lambda_{2}^{2}(\tau)\right)+\alpha\left(\frac{\lambda_{1}^{4} \lambda_{2}^{2}}{\lambda_{1}^{2}(\tau) \lambda_{2}^{2}(\tau)}-\lambda_{1}^{2}(\tau)\right)\right]\left[\lambda_{1}^{2}(\tau)-\frac{1}{\lambda_{1}^{2}(\tau) \lambda_{2}^{2}(\tau)}\right] \\
G_{3}^{\prime}= & \frac{\lambda_{1}}{\lambda_{2}}\left(\lambda_{2}-\lambda_{1}\right)\left(3+\lambda_{1}^{3} \lambda_{2}^{3}\right)+\alpha \lambda_{1}\left[\frac{3\left(\lambda_{2}-\lambda_{1}\right)}{\lambda_{2}}\left(\lambda_{1}^{2}(\tau)+\lambda_{2}^{2}(\tau)\right)+\lambda_{1}^{3} \lambda_{2}^{3}\left(\lambda_{1}^{2}(\tau)+\frac{1}{\lambda_{1}^{2}(\tau) \lambda_{2}^{2}(\tau)}\right)\right. \\
& \left.-\lambda_{1}^{4} \lambda_{2}^{2}\left(\lambda_{2}^{2}(\tau)+\frac{1}{\lambda_{1}^{2}(\tau) \lambda_{2}^{2}(\tau)}\right)-2 \frac{\left(\lambda_{1}(\tau)-\lambda_{2}(\tau)\right)}{\lambda_{2}(\tau)}\left(\lambda_{1}^{4} \lambda_{2}^{2} \lambda_{2}^{2}(\tau)-\frac{\lambda_{1}^{4} \lambda_{2}^{2}}{\lambda_{1}^{2}(\tau) \lambda_{2}^{2}(\tau)}-\lambda_{2}^{2}(\tau)\right)\right] \\
G_{3}^{\prime \prime}= & -2 \frac{\lambda_{1}}{\lambda_{2}}\left[\lambda_{1}(\tau)-\lambda_{2}(\tau)\right]\left[\lambda_{2}^{2}(\tau)-\frac{1}{\lambda_{1}^{2}(\tau) \lambda_{2}^{2}(\tau)}\right]\left[\left(\lambda_{1}^{4} \lambda_{2}^{2}-1\right)\left(1+\alpha \lambda_{2}^{2}(\tau)\right)\right. \\
& \left.+\alpha\left(\frac{\lambda_{1}^{4} \lambda_{2}^{2}}{\lambda_{1}^{2}(\tau) \lambda_{2}^{2}(\tau)}-\lambda_{1}^{2}(\tau)\right)\right]
\end{aligned}
$$

\section{NUMERICAL METHOD}

The numerical procedure for solving (2.5), (2.12) consists of two main parts. First, the Lee and Rogers method of linear viscoelasticity [11] is used to solve for $\partial \lambda_{1} / \partial r$ at each time step in terms of $\lambda$ and the past solution. The resultant system of differential equations is then solved in the elastic case.

Let the interval $[0, t]$ be partitioned by $n$ times $\left[t_{1}=0, t_{2}, \ldots, t_{n}=t\right]$. By $(3.5)$, the first integral in (2.12) can be written as

$$
\int_{0}^{t} G_{1}(\lambda ; \lambda(\tau), t-\tau) \mathrm{d} \tau=\int_{t_{1}}^{t_{n}} \partial_{t} C\left[I(\tau), \iota_{n}-\tau\right] \bar{G}_{1}\left(\lambda\left(t_{n}\right), \lambda(\tau)\right) \mathrm{d} \tau,
$$

where the definition of $\bar{G}_{1}$ in terms of $\lambda(t)$ and $\lambda(\tau)$ is obvious. Expressing (4.1) as a summation of $n-1$ integrals over the subintervals $\left(t_{k}, t_{k+1}\right),(k=1, n-1)$ and approximating each of these by the trapezoidal rule, one obtains

$$
\begin{aligned}
& \int_{0}^{t} G_{1}(\lambda ; \lambda(\tau) ; t-\tau) \mathrm{d} \tau \approx \frac{1}{2} \sum_{k=1}^{n-1}\left\{\left.\bar{G}_{1}\left(\lambda\left(t_{n}\right), \lambda\left(t_{k+1}\right)\right) \partial_{l} C\left[I(\tau), t_{n}-\tau\right]\right|_{\tau=t_{k+1}}\right. \\
& \left.+\left.\bar{G}_{1}\left(\lambda\left(t_{n}\right), \lambda\left(t_{k}\right)\right) \partial_{t} C\left[I(\tau), t_{n}-\tau\right]\right|_{\tau=t_{k}}\right\}\left(t_{k+1}-t_{k}\right) .
\end{aligned}
$$

Approximating the partial derivatives at $\tau=t_{k+1}$ and $\tau=t_{k}$ by backward and forward difference expressions, respectively, and evaluating the argument $I(\tau)$ at the appropriate points, (4.2) becomes

$$
\begin{aligned}
& \int_{0}^{t} G_{1}(\lambda ; \lambda(\tau) ; t-\tau) \mathrm{d} \tau \approx-\frac{1}{2} \sum_{k=1}^{n-1}\left\{\bar{G}_{1}\left[\lambda\left(t_{n}\right), \lambda\left(t_{k+1}\right)\right]\left(C\left[I\left(t_{k+1}\right), t_{n}-t_{k+1}\right]-C\left[I\left(t_{k+1}\right), t_{n}-t_{k}\right]\right)\right. \\
& \left.\quad+\bar{G}_{1}\left[\lambda\left(t_{n}\right), \lambda\left(t_{k}\right)\right]\left(C\left[I\left(t_{k}\right), t_{n}-t_{k+1}\right]-C\left[I\left(t_{k}\right), t_{n}-t_{k}\right]\right)\right\} .
\end{aligned}
$$


Upon rearranging terms in the summation, one obtains

$$
\begin{aligned}
-2 \int_{0}^{t} G_{1}(\lambda ; \lambda(\tau) ; t-\tau) \mathrm{d} \tau \approx & \bar{G}_{1}\left[\lambda\left(t_{n}\right), \lambda\left(t_{1}\right)\right]\left(C\left[I\left(t_{1}\right), t_{n}-t_{2}\right]-C\left[I\left(t_{1}\right), t_{n}-t_{1}\right]\right) \\
& +\bar{G}_{1}\left[\lambda\left(t_{n}\right), \lambda\left(t_{n}\right)\right]\left(C\left[I\left(t_{n}\right), 0\right]-C\left[I\left(t_{n}\right), t_{n}-t_{n-1}\right]\right) \\
& +\sum_{k=2}^{n-1} \bar{G}_{1}\left[\lambda\left(t_{n}\right), \lambda\left(t_{k}\right)\right]\left(C\left[I\left(t_{k}\right), t_{n}-t_{k+1}\right]-C\left[I\left(t_{k}\right), t_{n}-t_{k-1}\right]\right)
\end{aligned}
$$

It is seen from (3.5) that the last two integrals of (2.12) can also be written as in (4.1), but with an additional term involving $\partial C / \partial I$. This is differentiated analytically. However, the second differentiation with respect to $(t-\tau)$ is carried out numerically so as to eliminate factors $\left(t_{k+1}-t_{k}\right)$ and obtain finite sum approximations of form (4.4). In the finite sum approximations to the second integral, the term depending on $\lambda\left(t_{k}\right)$ has $\partial \lambda_{1} / \partial r\left(t_{k}\right)$ as a coefficient. For $t_{k}<t_{n}$, this derivative is approximated by a simple forward difference expression. For notational convenience, the finite sum approximations to the first and third integrals of (2.14) are denoted by $\sum_{1}$ and $\sum_{3}$, respectively. Let $\sum_{2}^{\prime}$ denote only the terms in the approximation to the second integral which contain $\lambda\left(t_{k}\right), k<n$, and $\partial \lambda_{1} / \partial r\left(t_{n}\right) G_{2}\left[\lambda\left(t_{n}\right)\right]$ denote the remaining term. It is convenient to note at this point that one also obtains expressions of type (4.4) in the finite sum approximations to the integrals in the stress expressions (3.4).

With these preliminaries out of the way, the details of solution can now be discussed. From (2.5) and (2.12) the initial elastic response, $t_{n}=t_{1}=0$, satisfies the following system of equations,

$$
\begin{aligned}
& \frac{\partial \lambda_{1}}{\partial r}\left(t_{1}\right)=\frac{1}{r} \frac{F_{2}\left(\lambda\left(t_{1}\right)\right)}{F_{1}\left(\lambda\left(t_{1}\right)\right)}, \\
& \frac{\partial \lambda_{2}}{\partial r}\left(t_{1}\right)=\frac{\lambda_{1}\left(t_{1}\right)-\lambda_{2}\left(t_{1}\right)}{r} .
\end{aligned}
$$

This system can be integrated by the Runga-Kutta method [12] subject to condition (2.13) at $\rho=1$. The specific value of $\lambda_{2}\left(1, t_{1}\right)$ is determined so that $\lambda_{2}\left(b, t_{1}\right)$ or $\rho \lambda_{3} \sigma_{1}$ at $(r, t)=\left(b, t_{1}\right)$ have the prescribed value, depending on whether a relaxation or creep problem is being considered. Since the correct value for $\lambda_{2}\left(1, t_{1}\right)$ is not known in advance, it is determined by a "shooting method", $\lambda_{2}\left(1, t_{1}\right)$ is guessed, $\lambda_{1}\left(1, t_{1}\right)$ is evaluated according to (2.13), (4.5) is integrated and the appropriate condition at $\rho=b$, say $\lambda_{2}\left(b, t_{1}\right)$, is evaluated. If $\left|\lambda_{2}\left(b, t_{1}\right)-\lambda^{*}\left(t_{1}\right)\right|>\varepsilon$, for some prescribed $\varepsilon, \lambda_{2}(b, t)$ is incremented and the process is repeated. This procedure is continued, using linear interpolation of the two most recent values to determine the next estimate of $\lambda_{2}\left(1, t_{1}\right)$.

For $n \geq 2,(2.12)$ is written in terms of the notation defined following (4.5) as

$$
\frac{\partial \lambda_{1}}{\partial r}\left(t_{n}\right)\left\{F_{1}\left(\lambda\left(t_{n}\right)\right)+\sum_{1}\right\}+\sum_{2}^{\prime}+\frac{\partial \lambda_{1}}{\partial r}\left(t_{n}\right) G_{2}\left[\lambda\left(t_{n}\right)\right]=\frac{1}{r}\left\{F_{2}\left(\lambda\left(t_{n}\right)\right)+\sum_{3}\right\} \text {. }
$$

Noting that $\partial \lambda_{1} / \partial r\left(t_{n}\right)$ can now be found explicitly, system (2.5), (2.12) is written as

$$
\begin{aligned}
\frac{\partial \lambda_{1}}{\partial r}\left(r, t_{n}\right) & =\frac{(1 / r)\left(F_{2}\left(\lambda\left(t_{n}\right)\right)+\sum_{3}\right)-\sum_{2}^{\prime}}{F_{1}\left(\lambda\left(t_{n}\right)\right)+\sum_{1}+G_{2}\left(\lambda\left(t_{n}\right)\right)}, \\
\frac{\partial \lambda_{2}}{\partial r}\left(t_{n}\right) & =\frac{\lambda_{1}\left(t_{n}\right)-\lambda_{2}\left(t_{n}\right)}{r}
\end{aligned}
$$


In the first equation of $(4.7), F_{1}, F_{2}$ and $G_{2}$ depend only on $\lambda\left(t_{n}\right)$, while $\sum_{1}, \sum_{2}^{\prime}$ and $\sum_{3}$ depend on $\lambda\left(t_{n}\right)$ and $\lambda\left(t_{k}\right), k<n$. Because the latter have been found by solution of $(4.7)$ for times $t_{k}<t_{n}, \sum_{1}, \sum_{2}^{\prime}$ and $\sum_{3}$ may now be considered functions of the independent variable $r$ and $\lambda\left(t_{n}\right)$. Thus, for each time $t_{n}$, the stretch ratios are found by solving a coupled system of nonlinear ordinary differential equations. The solution of (4.7), subject to appropriate boundary conditions at $\rho=1$ and $\rho=b$, is obtained by the same procedure as was outlined for the initial elastic response. The value of $\lambda_{2}\left(1, t_{k}\right)$ which gives acceptable satisfaction of the boundary condition is the first guess at time $t_{k+1}$.

Once the stretch ratios have been calculated, the radial deformation function $\rho(r, t)$ is obtained directly from the second of (2.2), while stresses are calculated from the above mentioned approximate expressions for (3.4).

Since the summations in the right hand side of (4.7) must be recalculated at each time step and since their summands are functions of $\lambda$ which are evaluated at each iteration for finding $\lambda_{2}\left(1, t_{k}\right)$ and several times during the Runga-Kutta procedure, computation time must be considered. From (3.4) and (3.5), it is seen that the integrands of the stress integrals and $G_{1}, G_{2}^{\prime}, G_{2}^{\prime \prime}, G_{3}^{\prime}, G_{3}^{\prime \prime}$ are products of functions of $\lambda$ with functions of $\lambda(\tau)$. Consequently, the right hand side of (4.7) can be written in terms of products of functions of $\lambda$ with summations involving $\lambda\left(t_{k}\right), k=1, n$. As in (4.4) these summations consist of a partial sum involving the previously determined $\lambda\left(t_{k}\right), k<n$ and one term involving the current unknown $\lambda\left(t_{n}\right)$. At each time step these partial sums were calculated, stored and treated as radially dependent coefficients in (4.7).

One useful means of checking the accuracy of solution of system (2.5), (2.12) is that the residual elastic solution can be calculated directly. Assuming that the stretch ratios reach a steady state value as $t \rightarrow \infty,(2.12)$ and (3.4) can be integrated for large $t$ to give, together with (2.5), the governing equations. The direct solutions of this system can then be compared with the large time limit of the transient solution. For the material model considered, the residual elasticity equations for $\lambda_{1}, \lambda_{2}$ are exactly the same as the initial elastic equations (4.5). Thus, if $\lambda_{2}(b, t)=\lambda^{*}(t)$ is constant in the relaxation problem, the residual solution coincides with the initial solution. In the creep problem, where $\sigma_{1}(b, t)$ enters the boundary condition, $C_{\infty}<C_{0}$ so the residual solution must be calculated separately.

\section{DISCUSSION}

All calculations were carried out for a membrane having an outer radius five times greater than the inner radius, i.e. $b / a=5$. The Mooney model parameter $\alpha$ was chosen as 0.1 , and the ratio of long term to initial moduli $C_{\infty} / C_{0}$ was $1 / 3$. For both the creep and the relaxation problem calculations were carried out for $\beta=0$ and $\beta=0.05$. For the former value, relaxation time is independent of stretch ratio. The latter value was chosen so that the stretch ratio dependent relaxation time $1 / p(I)$ defined by $(3.3)$ would be approximately $\tau_{R} / 2$ when $\lambda_{2} \approx 4$ at the inner hole $(r=1)$ where the most severe stretching occurs. For the creep problem, detailed calculations were carried out for $F^{*}(t)=3 \cdot 0$, and for the relaxation problem, $\lambda^{*}(t)=1.5$. At each time step, the value of $\lambda_{2}(1, t)$ was accepted when $\left|\lambda_{2}(5, t)-\lambda^{*}\right|<\varepsilon$ or $\left|\rho \lambda_{3} \sigma_{1}\right|_{r=5}-F^{*} \mid<\varepsilon$, where $\varepsilon=0.001$. In general, for this choice of $\varepsilon$, three or four iterations were required at each time step to arrive at an acceptable value for $\lambda_{2}(1, t)$. A smaller value of $\varepsilon$ was not chosen because of increased computation time. For $\varepsilon=0.001$ and the chosen radial increment and time steps, the creep problem required 
somewhat more than two minutes of computation time. Computations were carried out on the IBM 360 computer at the University of Michigan Computing Center.

The determination of time steps and radius increments was carried out for the creep problem with $F^{*}(t)=3.0$, this having the more complicated boundary condition at $r=5$ and for $\beta=0.05$, this causing faster changes in stretch ratios due to smaller relaxation times. The experience of Lee and Rogers [10] in solving integral equations in linear viscoelasticity suggests that time steps $t_{k}$ should vary logarithmically. This permits small time increments for early times when quantities are undergoing large variation and gives larger time increments for later times when variations are smaller. The time steps finally used were given by the relation $t_{k+1}=t_{k} 10^{A}$, where $A=0.2$ for $k=2,10$ (up to 0.63 ), $A=0.05$ for $k \geq 11$, with $t_{2}=0.01$. Changing $t_{2}$ to 0.001 caused a maximum stretch ratio change of 0.0001 at $t=0.01$, a change of less than 0.02 per cent which was considered insignificant. A refinement of the first set of time steps to $A=0 \cdot 1, k=2,20$ (up to $t=0.63$ ), caused a maximum stretch ratio change in the fifth decimal place and stress change in the fourth decimal place, again considered negligible. A smaller value of $A$ for $k>11$ was necessary because time increments became too large. The same computation with $A=0.025$, $11 \leq k \leq 45$ (up to $t=5.01$ ), $A=0.05,46 \leq k<55$, produced maximum changes in the stretch ratio of less than 0.5 per cent and in stresses of less than 1 per cent. This slight improvement in accuracy was considered offset by the increase in computation time due to the increased number of time steps.

The numerical procedure turned out to be more sensitive to the choice of the radial increment $d$ than to the choice of time increments. Since the Runga-Kutta procedure for integrating (4.7) has an error of $0\left(d^{5}\right)$, a choice of $d=0.2$ is quite adequate for obtaining the initial solution or directly calculating the residual solution. In fact, changing from $d=0.2$ to $d=0.1$ caused at most a fourth decimal place change in the stretch ratios or stresses. The choice of $d=0.2$ is not adequate at intermediate times, however, because the radial dependent coefficients on the right hand side of (4.7) are found numerically and depend on the earlier solutions. Hence, $d$ must be smaller than 0.2 in order to ensure accurate calculation of these coefficients and therefore an accurately defined differential equation at each time step. With the additional realization that stress concentration near the hole will cause large stretch gradients, it was finally decided to choose $d=0.05$, $1 \leq r \leq 3, d=0 \cdot 1,3 \leq r \leq 5$. Changing from $d=0.2$ to $d=0 \cdot 1,1 \leq r \leq 5$, in obtaining the transient solution produced changes in stretch ratios and stress of up to 5 per cent. A change from $d=0.1,1 \leq r \leq 5$, to the final choice caused a maximum change in stretch ratios of 1.8 per cent and in stresses of 3 per cent. These changes occurred near the hole and rapidly decreased to about 0.5 per cent as $r$ increased to the outer boundary. From a numerical point of view this accuracy could be improved by further reducing $d$, especially near the hole and increasing the number of time steps. From an engineering point of view, since this causes a substantial increase in computation time, the present accuracy was considered satisfactory.

Confidence in the accuracy of the numerical method for the above stated choices of radial and time increments is enhanced by considering the relaxation problem. As was mentioned at the end of Section 4, the initial and residual solutions for the relaxation problem should coincide. In fact, the residual solution limit of the transient solution differed from the initial state by at most 0.5 per cent in the stretch ratios and 1 per cent in the stresses. Furthermore, the assumed constitutive equation is such that when $\beta=0$, the stretch ratios are independent of time. That is, the assumption $\lambda_{i}(r, t)=\lambda_{i}(r)$ implies 
that $\sigma_{i}(r, t)=\sigma_{i}(r) f(t)$. Since $f(t)$ drops out of $(2.8)$ this solution is possible. The numerical solution of (2.5), (2.12) for $\lambda^{*}(t)=1.5, \beta=0.0$, does not differ from the initial state until $t=3.16$ and then the stretch ratios change by at most 0.4 per cent to a new value which does not vary with time.

As a final comment on the accuracy of the numerical procedure it is useful to point out that near the hole $\lambda_{2}>1$ and $\lambda_{1}<1, \lambda_{3}<1$, so that, by (3.4), $\sigma_{2}$ depends primarily on $\lambda_{2}^{2}$. If the numerical solution gives $\lambda_{2}(1+\varepsilon)$, where $\varepsilon$ is a small error term, then $\sigma_{2}$ depends on $\lambda_{2}^{2}(1+2 \varepsilon)$. Thus, the change in stress is usually about twice the change in stretch ratio.

The numerical solution to the relaxation problem is represented in Figs. 24. As was mentioned above, when $\beta=0$, the kinematic quantities $\rho / a, \lambda_{1}$ and $\lambda_{2}$ do not vary with time. Their distribution is shown in Fig. 2. When $\beta=0.05$, these quantities vary only slightly from their initial values, or equivalently, from the solution with $\beta=0$. Hence Fig. 2 can also be considered to show their initial values. When $\beta=0.05, r$ and $t$ are coupled in the relaxation function through the product $p(I(r)) t$. Figure 2 shows that $\tau_{\mathrm{R}} p(I(r))$ varies only slightly with $r$ so that this coupling is weak. Thus, the results that $\lambda_{i}(r, t) \approx \lambda_{i}(r)$ is a consequence of the fact that by (3.4) one also has that $\sigma_{i} \approx \sigma_{i}(r) g(r, t)$ and the variation of $g(r, t)$ with $r$ is small. Figures $2-4$ show the strong local influence of the hole. A moderate circumferential stretch ratio at $r=5, \lambda_{2}=1.5$, is magnified to $\lambda_{2}=2.85$ at $r=1$. The local influence of the hole is evidenced by the observation from Fig. 2 that at $r=5 \lambda_{\mathrm{I}}$ has already approached to within 10 per cent of $\lambda_{2}$. Since $\lambda_{2}>1$ and $\lambda_{1}=\lambda_{3}<1$ near $r=1$, it follows from (3.4) that $\sigma_{2}$ near $r=1$ depends primarily on $\lambda_{2}^{2}$. Thus, as Fig. 3 shows, the strain concentration generates a high stress concentration. Figures 3 and 4 show

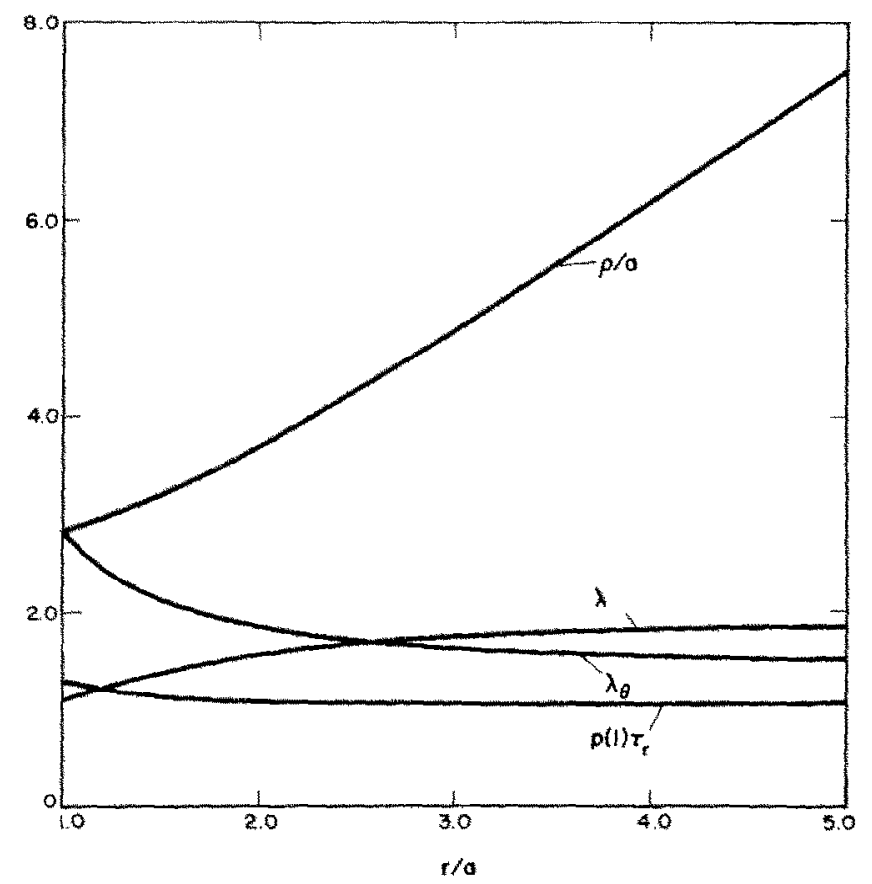

Fio. 2. Relaxation problem (stretch boundary condition)-particle distribution of radial mapping, stretch ratios and inverse of stretch ratio dependent relaxation time. 


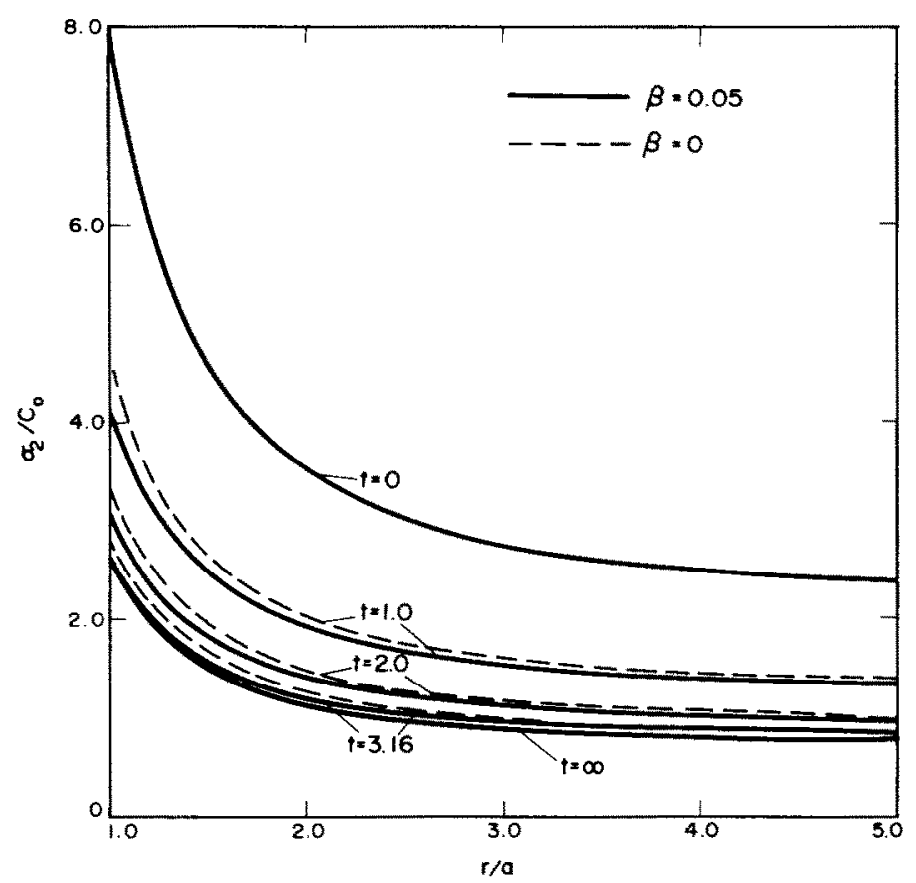

Frg. 3. Relaxation problem-particle distribution of circumferential stress $\sigma_{2} / C_{0}$ at various times for $\beta=0.0$ and $\beta=0.05$.

that the stress distribution corresponding to $\beta=0$ relaxes slower than the stress distribution for $\beta=0.05$. Furthermore, when $\beta=0.05$, the strain concentration causes the stress concentration to relax out faster than when $\beta=0$.

The deformation history of the membrane for the creep problem is shown in Fig. 5. As the solution for $\beta=0.05$ shows, dependence of relaxation time on the stretch ratios strongly influences how the membrane approaches its residual state. This follows from the fact that for the faster relaxing material, the strain at a particle increases more rapidly in order to balance the applied stresses. At $t=1.0$ the relaxation time away from the hole is about 5 per cent less than if $\beta=0$ and the membrane is closer to its residual deformed state. The relaxation time near the hole is about 20 per cent less than in the rest of the membrane. Since higher stresses are required near the hole, particles must stretch more and the hole opens more than if $\beta=0$. As the residual state is approached and time effects diminish, the particles approach residual elastic behavior and the membrane readjusts to the same final shape as for $\beta=0$.

The distribution of stress components with respect to membrane particles is shown in Figs. 6 and 7. Because of the large deformation of the membrane, it was more convenient to plot the stress against particle label $r / a$. When $\beta=0$, these stresses increase monotonically from their values in the initial elastic response state to their residual values. When $\beta=0.05$ these figures also show the significant changes in the stress histories and distributions arising from the membrane overshoot which results when relaxation time is decreased by the amount of stretching. 

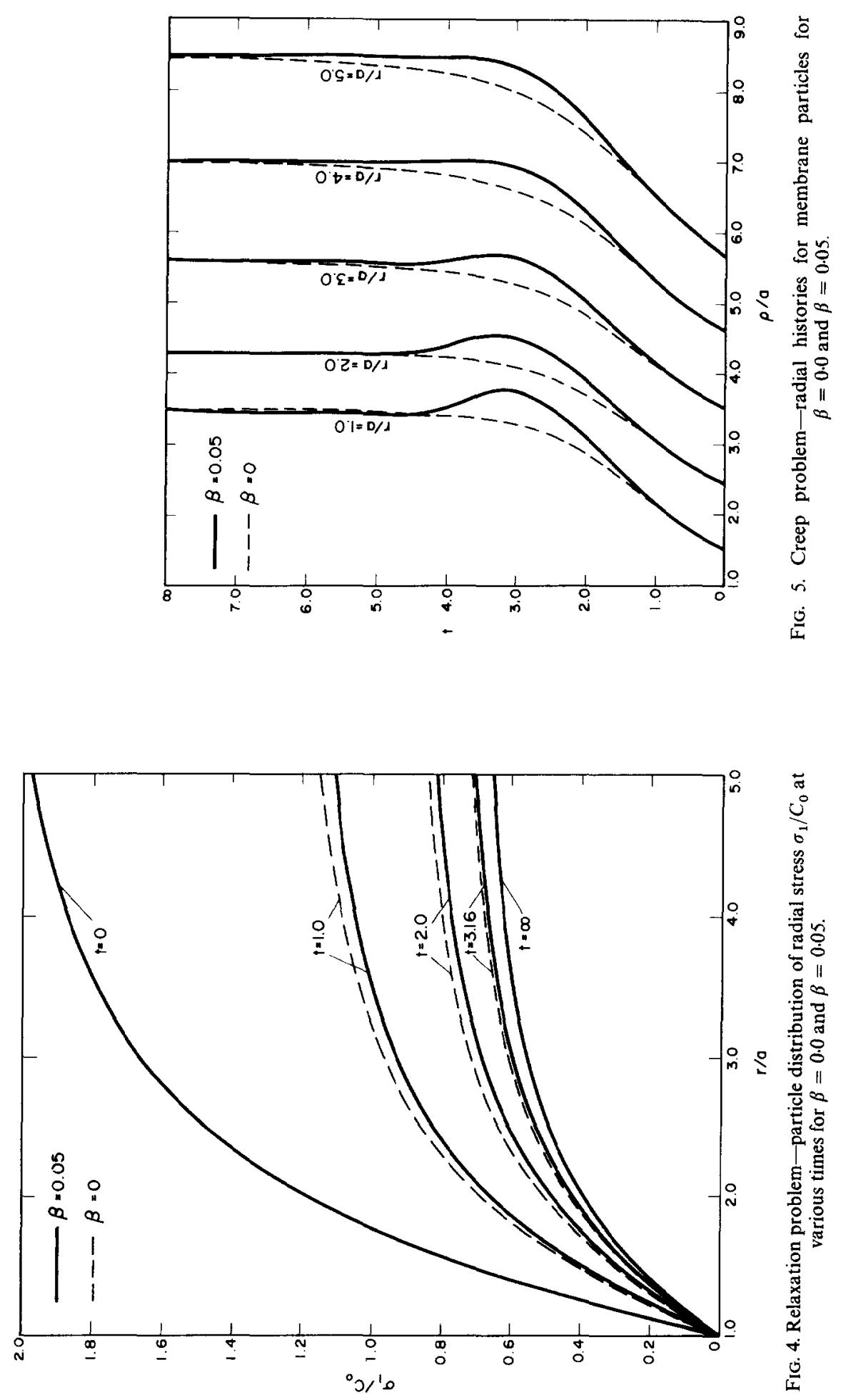

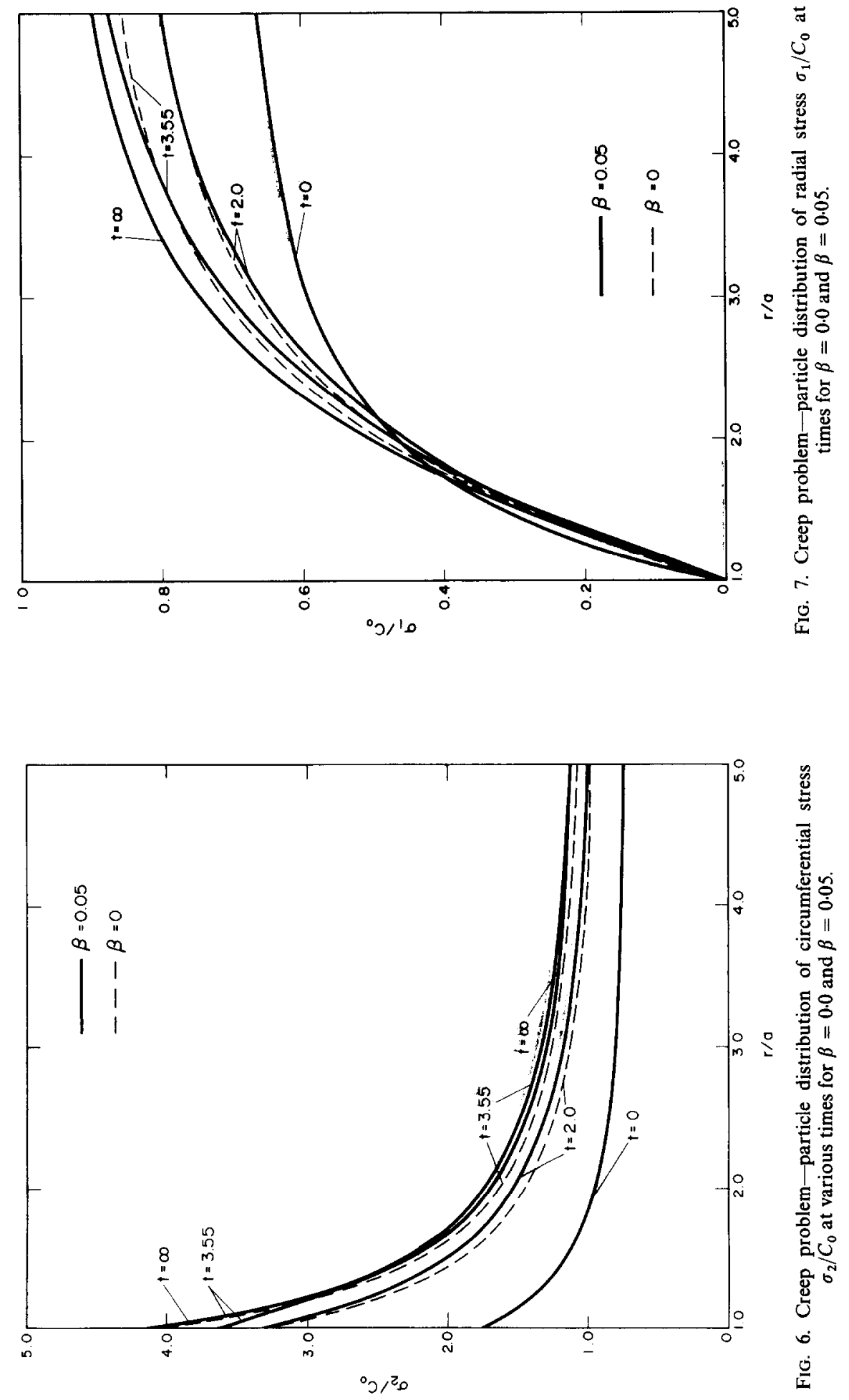
Acknowledgements-The author wishes to express his thanks to the Rackham Graduate School of the University of Michigan for their financial assistance under a Faculty Research Grant, to Mr. John Jin-Jau Shi for his computation assistance and to the Laboratium voor Technische Mechanica of the Technische Hogeschool, Delft.

\title{
REFERENCES
}

[1] W. H. YANG, Stress concentration in a rubber sheet under axially symmetric stretching. J. appl. Mech. 34, 942 (1967).

[2] W. H. YANG and W. W. FenG, On axisymmetric deformations of nonlinear membranes. J. appl. Mech. 37. $1002(1970)$.

[3] W. H. YANG and K. H. HsU, Indentation of a circular membrane. J, appl. Mech. 38, 227 (1971).

[4] R. S. Rivlin and A. G. Thomas, Large elastic deformations of isotropic materials-VIII. Strain distribution around a hole in a sheet. Phil. Trans. R. Soc. A243, 289 (1951).

[5] B. D. Coleman and W. Noll, Foundations of linear viscoelasticity. Rev. Mod. Phys. 33, 239 (196I).

[6] A. C. Pipkin and T. G. Rogers, A nonlinear integral representation for viscoelastic behavior. J. Mech. Phys. Solids 16, 59 (1968).

[7] A. S. WINEMAN and A. C. PIPKIN, Material symmetry restrictions on constitutive equations. Archs ration. Mech. Analysis 17, 185 (1964).

[8] K. C. VAlanis and R. F. LANDEL, Large multi-axial behavior of a filled rubber. Trans. Soc. Rheo. 11, 243 (1967).

[9] S. Middleman, Transient response of an elastomer to large shearing and stretching deformations. Trans. Soc. Rheo. 13, 123 (1969).

[10] B. C. Bocue, An explicit constitutive equation based on an integrated strain history. Ind. Engng Chem. Fundamentals 5, 253 (1966).

[11] E. H. LEE and T. G. RoGERS, Solution of viscoelastic stress analysis problems using measured creep or relaxation functions. $J$. appl. Mech. 30,127 (1963).

[12] R. W. HAMming, Numerical Analysis for Scientists and Engineers, p. 212. McGraw-Hill (1962).

(Received 16 June 1971 ; revised 26 October 1971)

\begin{abstract}
Абстракт-Рещаются две задачи для больших плоских осесимметрических деформаций кольцевой мембраны, изготовленной из нелинейного, вязкоупругого материала. Одна задача касается заданной деформации на внешней транице, вторая же заданного усилия. Эти задачи подобраны в качестве примеров, для иллюстрации обобщения класса задач для вязкоупругой мембраны, на основе соответствующей задачи угругой мембраны, предложенной онгом, очень удобным для численного расчета. В формулировке используются коэффиниенты радиального окружного растяжения, в качестве зависимых переменных. В этом случае, они находятся путем решения нелинейных дифференциально-интетральных уравнений, первого порядка. Численный процесс является таким же, что при каждой очередной степени, задана соответствует системе нелинейных, обыкновенных дифференциальных уравнений, для трансформационных коэффициентов растяжения. Затем, такая же система интегрируется путем такого же числового процесса, как в соответствующей упругой задаче.
\end{abstract}

